\title{
Synergistic antitumor efficacy by combining adriamycin with recombinant human endostatin in an osteosarcoma model
}

\author{
HAIRONG XU ${ }^{1}$, XIAOHUI NIU ${ }^{1}$, QING ZHANG $^{1}$, LIN HAO $^{1}$, YI DING $^{1}$, WEIFENG LIU $^{1}$ and LU YAO $^{2}$ \\ ${ }^{1}$ Department of Orthopedic Oncology Surgery, Beijing Ji Shui Tan Hospital, Peking University; \\ ${ }^{2}$ Department of Pathology, School of Basic Medical Sciences, Peking University, Beijing 100035, P.R. China
}

Received October 12, 2010; Accepted May 10, 2011

DOI: $10.3892 / \mathrm{ol} .2011 .334$

\begin{abstract}
In the last 15 years, chemotherapy-based therapeutic regimens for the treatment of osteosarcoma have failed to demonstrate improved survival rates. Novel approaches, including targeted therapy and antiangiogenic therapy, may provide new methods for the treatment of osteosarcoma, one of the most deadly malignant diseases. In the present study, the therapeutic efficacy of an endogenous angiogenesis inhibitor, endostatin, was tested in combination with the chemotherapeutic agent, adriamycin. BALB/c mice, aged 4-6 weeks were fed animal chow and had access to water ad libitum. The mice were divided into groups and injected with tumor cells. Immunohistochemical staining was performed to identify the microvessel density. The TUNEL technique was also used to determine the apoptotic index. The combination of endostatin and adriamycin produced marked synergistic antitumor activity in a mouse osteosarcoma model. These findings provide new guidelines for designing future clinical trials and for the application of currently available clinical drugs (endostatin has been approved for clinical use) in the treatment of osteosarcoma.
\end{abstract}

\section{Introduction}

Osteosarcoma is the most common malignant bone tumor affecting children and adolescents $(1,2)$. The survival of patients with osteosarcoma has markedly improved from less than $20 \%$ reported in the 1960 s to approximately $65 \%$ over the past 30 years (3-6), largely as a result of chemotherapeutic advances. However, for the past 15 years, the survival rate has remained the same in countries including China (7), despite continuing advances in surgical techniques and aggressive combination chemotherapy $(4,8,9)$. Furthermore, chemotherapeutic agents against osteosarcoma have highly toxic effects and it appears that the doses of conventional cyto-

Correspondence to: Professor Xiaohui Niu, Department of Orthopedic Oncology Surgery, Beijing Ji Shui Tan Hospital, Peking University, Beijing 100035, P.R. China

E-mail: niuxiaohui@263.net

Key words: osteosarcoma, antiangiogenic therapy, combination therapy, synergistic effect toxic agents have been maximally utilized in osteosarcoma (4). Second-line therapy for metastatic or recurrent osteosarcoma continues to present a challenge. Therefore, alternative treatment modalities for osteosarcoma should be identified and utilized.

One promising emerging therapy involves antiangiogenic treatment. This treatment strategy aims to suppress tumor growth through the inhibition of tumor angiogenesis (10). Findings of studies have shown that when tumors are deprived of new blood vessels they remain in a microscopic state of dormancy (11). A number of antiangiogenic agents are under evaluation in preclinical and clinical trials (12). Endostar ${ }^{\mathrm{TM}}$, a novel recombinant human endostatin expressed and purified in Escherichia coli with an additional nine-amino acid sequence forming another his-tag structure, was approved by the State Food and Drug Administration of China (SFDA) in 2005 for the treatment of non-small cell lung cancer. Since antiangiogenic drugs are directed against developing vasculature, but not tumor cells, they may achieve stable disease, as opposed to partial response or complete remission (13). Moreover, discontinuation of antiangiogenic therapy may allow a tumor to resume growth. Thus, antiangiogenic treatment alone is not suitable for patients with malignant tumors.

Combination cytotoxic agents with antiangiogenic compounds may lead to improved antitumor efficacy by targeting both tumor and endothelial cell compartments $(14,15)$. This use of antiangiogenic agents in combination with cytotoxic therapy for solid tumors has been supported in numerous in vivo studies (16-18). Strategies combining the two agents have not, however, been investigated in nude micebearing human osteosarcoma cells.

In this study, the efficacy of combinations of adriamycin and rh-endostatin was assessed in an osteosarcoma nude mouse model. The effects of combination therapy were compared with those of adriamycin and antiangiogenic agents alone.

\section{Materials and methods}

Drugs. Recombinant human endostatin (Endostar ${ }^{\mathrm{TM}}$ ) was kindly supplied by Jiangsu Simcere Pharmaceutical R\&D (Nanjing, Jiangsu, China). Endostar was administered by daily intraperitoneal (IP) injections of $2.5,5.0$ or $10 \mathrm{mg} / \mathrm{kg}$ body weight. The control group received the physiological saline $(20 \mathrm{mg} / \mathrm{kg})$ daily via IP injection. 
Adriamycin (Pharmacia Italia SPA, Group of Pfizer, Italy) was used for chemotherapy, in doses of 2.5 and $5 \mathrm{mg} /$ $\mathrm{kg}$ body weight, and was administered intraperitoneally every 4 days. The control group received the same as the rh-endostatin group.

Tumor cell line. Human osteosarcoma cell line OS-732 was purchased from the Orthopaedics Graduate School in Beijing Ji Shui Tan Hospital and cultured in RPMI-1640 (Solarbio Science and Technology, Beijing, China) supplemented with $10 \%$ FCS (Sino-US Lanzhou Biological Engineering, Shanghai, China) and $1 \%$ glutamine-penicillin-streptomycin. The cells were incubated in a humidified atmosphere of 5\% $\mathrm{CO}_{2}$ at $37^{\circ} \mathrm{C}$. For tumor cell injection, the cells were grown in $900 \mathrm{~cm}^{2}$ roller bottles.

Animals. Female BALB/c mice, aged 4-6 weeks, were purchased from the Institute of Laboratory Animal Sciences, Peking Union Medical College. Mice were acclimated, caged in groups of five in a barrier care facility, and fed with animal chow and water ad libitum. The experiments were carried out according to the guidelines of the Animal Welfare Committee of Peking University Health Science Center.

Tumor cell transplant. Cells to be xenotransplanted were washed in phosphate-buffered saline (PBS) buffer, resuspended in serum-free culture medium, counted in a hemocytometer and adjusted to a density of $1 \times 10^{7}$ cells $/ \mathrm{ml}$. Mice were shaved and the dorsal skin was cleaned with ethanol prior to tumor cell injection. A suspension of $2 \times 10^{7}$ tumor cells in $0.2 \mathrm{ml}$ RPMI-1640 was injected into the SC dorsa of mice at the proximal midline.

Animals with OS-732 osteosarcoma were sacrificed, and the skin overlying the tumor was cleaned with Betadine and ethanol. In a laminar flow hood, tumor tissue was excised under aseptic conditions. A suspension of tumor cells in $0.9 \%$ saline was made by passage of viable tumor tissue through a sieve and a series of sequentially smaller hypodermic needles of diameter 22- to 30-gauge. The final concentration was adjusted to $1 \times 10^{7}$ cells $/ \mathrm{ml}$, and the suspension was placed on ice. The injection of tumor cells was performed as described above.

Methods. The treatment was initiated 12 days after tumor cell injection when the mean tumor volume was $215 \mathrm{~mm}^{3}$. Nude mice bearing established OS-732 human osteosarcoma were divided into 7 groups of 7 , with the exception of the lowdose rh-endostatin group $(n=6)$. The groups received varying doses of rh-endostatin or adriamycin alone as well as combinations of the two agents. For single-agent antiangiogenic therapy, the varying doses of rh-endostatin were high-dose $(10 \mathrm{mg} / \mathrm{kg})$, middle-dose $(5 \mathrm{mg} / \mathrm{kg})$ and low-dose $(2.5 \mathrm{mg} / \mathrm{kg})$. The single-agent chemotherapy treatment group received $5 \mathrm{mg} / \mathrm{kg}$ weight of adriamycin. Combination therapies involved treatment with middle- or low-dose rh-endostatin combined with adriamycin.

The mice were weighed every 3 days by an investigator who did not know the treatment schedule. Tumors were weighed and fixed in buffered Formaldehyde-Fresh (Fisher Scientific, Fair Lawn, NJ, USA) after the mice were sacrificed.
Histopathologic examination. Small samples $\left(2-3 \mathrm{~cm}^{3}\right)$ of tissue were fixed in $10 \%$ buffered formalin and embedded in paraffin. Paraffin-embedded blocks of tissue were sectioned at $5 \mu \mathrm{m}$, deparaffinized, and stained with hematoxylin and eosin (H\&E). The slides were examined under a light microscope.

Immunohistochemistry. To identify the microvessel density (MVD), immunohistochemical staining was performed using monoclonal antibodies to rabbit anti-Fac VIII (intratumoral vascular endothelium) (Zymed Laboratories, South San Francisco, CA, USA). Briefly, deparaffinized tissue sections were incubated with a primary antibodies after being treated with $3 \%$ hydrogen peroxide and processed for antigen retrieval by heating in a microwave oven at $96^{\circ} \mathrm{C}$, in $0.01 \mathrm{M}$ citrate buffer $\mathrm{pH} 6$, for $15 \mathrm{~min}$. The reaction products were colorized with PV9000 immunohistochemistry (IHC) kit (Zymed Labs) and DAB substrate-chromogen kit, resulting in a brown signal. Negative controls were carried out with PBS instead of the primary antibody. The vessel density was identified according to the procedures described by Weidner (19), at low magnification (x40-100). Regions with the highest vessel density ('hot-spot' regions) were scanned and counted at a magnification of x200 (0.738 $\mathrm{mm}^{2}$ field) by an observer who did not know the treatment schedule. At least five fields were counted in a representative tumor section, and the highest three counts were recorded.

Determination of the apoptotic index. The terminal deoxynucleotidyl transferase-mediated deoxyuridine triphosphate nick end-labeling (TUNEL) technique served as a marker of tumor cell apoptosis and was carried out in accordance with the manufacturer's instructions (Roche Diagnostics, Penzberg, Germany). In brief, the sections were deparaffinized with xylene and incubated with primary antibodies after being treated with $3 \%$ hydrogen peroxide and processed for antigen retrieval by heating in a microwave oven at $96^{\circ} \mathrm{C}$, in $0.01 \mathrm{M}$ citrate buffer $\mathrm{pH} 6.0$, for $15 \mathrm{~min}$. End-labeling was achieved by catalytically adding residues of digoxygeninlabeled 11-dUTP and dATP to the 3'-hydroxyl ends of DNA with the enzyme TdT. The reaction buffer containing dUTP, dATP and TdT was applied for $60 \mathrm{~min}$ at $37^{\circ} \mathrm{C}$ in a humidified atmosphere. Negative controls were carried out with PBS. The digoxygenin was detected immunohistochemically with a digoxygenin-specific peroxidase-conjugated antibody (30 min in a humidified atmosphere at room temperature). For the color reaction, a DAB substrate-chromogen kit was used, resulting in a brown signal. The sections were counterstained with hematoxylin. Tumor cells in non-necrotic tumor areas with detectable staining of the nucleus were regarded as cells undergoing apoptosis. The apoptotic index (AI) was determined as the number of apoptotic tumor cells per section divided by the total number of tumor cells per section (20).

Statistical analysis. Statistical analyses were performed using SPSS 15.0 software. For differences in tumor weight, MVD and AI, as well as ANOVA were used for statistical analysis. Results are mean \pm SD. The p-values were two-sided and statistical significance was defined as $\mathrm{p}<0.05$.

The antitumor activity of the combination therapy was analyzed by the fractional product method as previously 

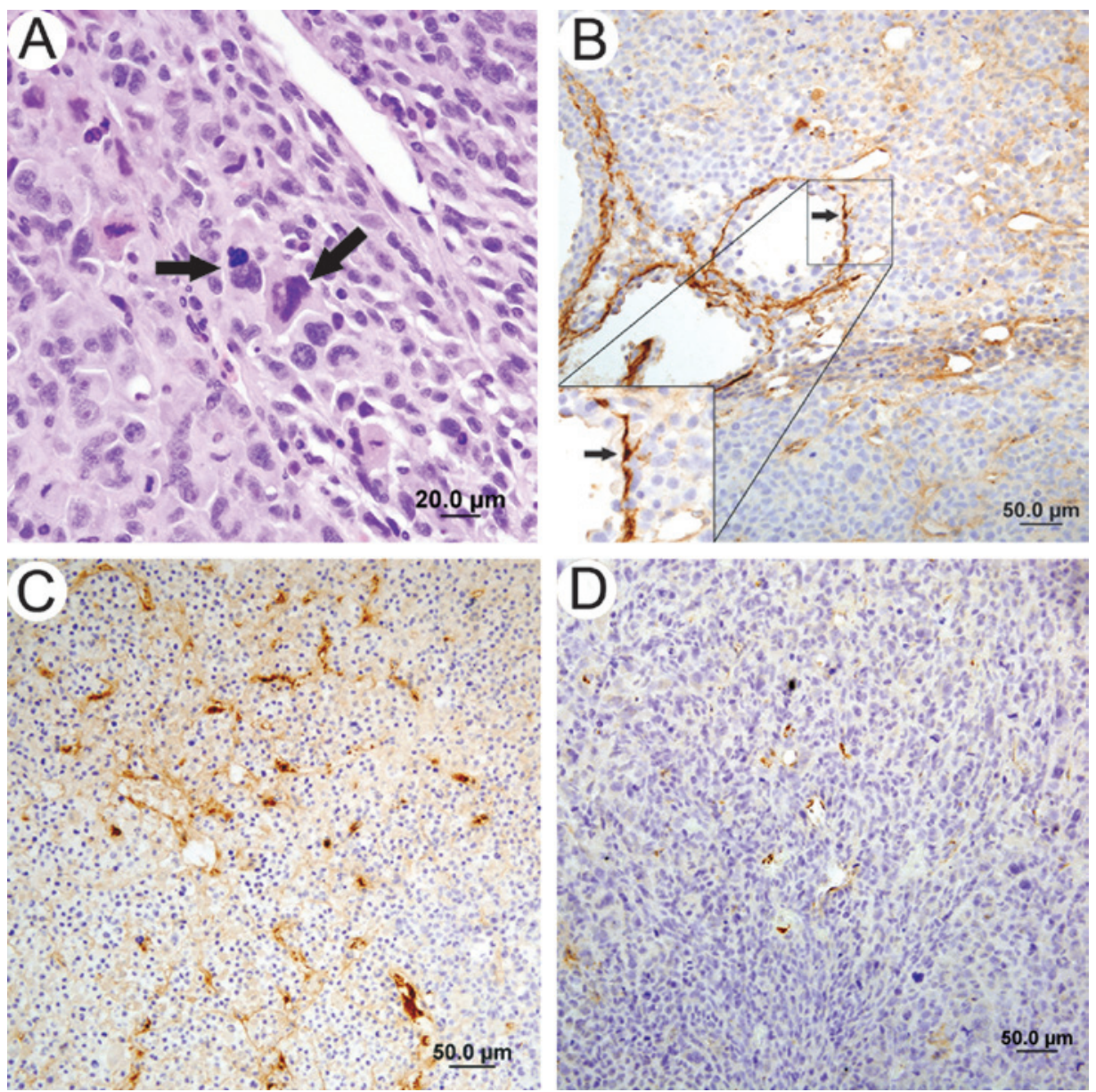

Figure 1. (A) Tissue stained with hematoxylin and eosin is shown and the nuclear pleomorphism of malignant tumor feature is evident (arrow). (B) Intratumoral vascular endothelium cells were stained by rabbit anti-Fac VIII (arrow). Tumor tissue with (D) high-dose rh-endostatin treatment alone showed fewer new vascular endothelium cells than the (C) control. (B-D) were counterstained with hematoxylin.

described (21). Fractional tumor weight (FTW) relative to controls was calculated as the ratio between the mean tumor weight of the experimental groups and the mean tumor weight of the control group. The expected FTW of the combination is the product of the mean FTW of the two agents given as single drugs. The ratio was obtained by dividing the expected FTW by the observed FTW of the combination. A ratio of $>1$ indicates a synergistic effect, and a ratio of $<1$ indicates a less than additive effect.

\section{Results}

Animal survival. Since there was no objective simple evaluation system for mice, only descriptive results were employed in this study. Nude mice treated with adriamycin alone or in combination showed less weight gain than those treated with rh-endostatin alone or those in the control group. One mouse died in every group that included treatment with adriamycin before the end of the experiment.

Antitumor effect. With the rh-endostatin monotherapy, mice treated with any dose exhibited significant tumor inhibition compared to the controls (Table I). When compared with each other, the varied doses of rh-endostatin showed no significant difference in the monotheraphy groups.
Adriamycin monotherapy. In this pilot experiment, almost $35 \%$ of the mice treated with adriamycin $(5 \mathrm{mg} / \mathrm{kg})$ did not survive to the end of the experiment due to the toxic response to the cytotoxic agent (data not shown). A lower dose, also resulting in a significant antitumor effect but with acceptable toxicity, was determined. The tumor weight of this group was $2.58 \mathrm{~g}$ with a tumor inhibitory rate of $29.1 \%$.

Combination treatment. Treatment with low-dose rh-endostatin and adriamycin alone resulted in tumor growth inhibition at rates of 25.3 and $29.1 \%$, respectively. A combination of lowdose rh-endostatin and adriamycin showed greater antitumor activity at approximately $42.9 \%$, suggesting a less than additive effect (Table II). High-dose rh-endostatin alone showed a tumor growth inhibition of $34.1 \%$. However, a combination of high-dose rh-endostatin and adriamycin showed a synergistic antitumor effect with a tumor growth inhibition of approximately $64.8 \%$.

Microvessel density (MVD) counts. As shown in Table III and Fig. 1, there was a significant difference $(\mathrm{p}<0.01)$ in MVD between all treatment groups and the control group (Table III). The difference between the low-dose rh-endostatin combination treatment and rh-endostatin monotherapy was not significant $(p=0.317)$. The difference between the high-dose 

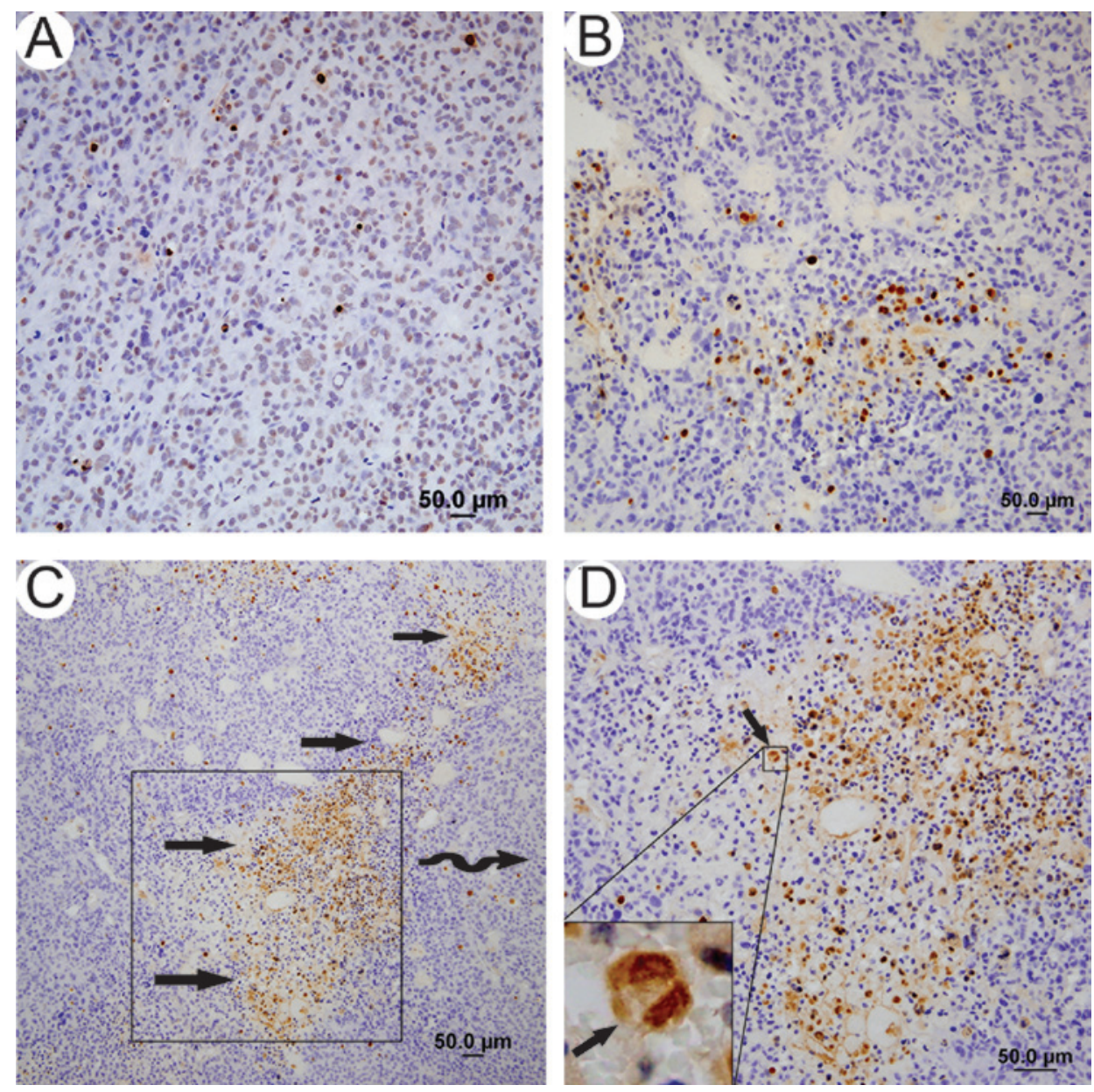

Figure 2. Combination treatment of high-dose (B) rh-endostatin and adriamycin showed more apoptotic tumor cells than the (A) control. (C) and (D) show that apoptotic foci were often concentrated within the perivascular areas in the combination treatment groups.

Table I. Tumor weight and tumor inhibition rate of various rh-endostatin treatment groups.

\begin{tabular}{lcc}
\hline & $\begin{array}{c}\text { Tumor weight } \\
( \pm \mathrm{s})(\mathrm{g})\end{array}$ & Tumor inhibition rate (\%) \\
\hline Control & $3.64 \pm 0.75$ & \\
Low-dose & $2.72 \pm 0.44$ & $25.3^{\mathrm{a}}$ \\
Middle-dose & $2.40 \pm 0.36$ & $34.1^{\mathrm{a}}$ \\
High-dose & $2.34 \pm 0.34$ & $35.7^{\mathrm{a}}$ \\
\hline
\end{tabular}

${ }^{\mathrm{a}} \mathrm{p}<0.01$.

rh-endostatin combination treatment and rh-endostatin monotherapy showed marginal significance $(\mathrm{p}=0.05)$.

Apoptotic index. The AI was significantly higher in the treatment groups compared with the control group, with the exception of the low- and middle-dose rh-endostatin groups (Table IV, Fig. 2A and B). The high-dose rh-endostatin combination treatment showed a significantly higher AI than adriamycin alone, which was not found for the low-dose rh-endostatin combination treatment. In addition, apoptotic foci were often concentrated within the perivascular areas in the combination treatment groups (Fig. 2C and D).

\section{Discussion}

Conventional chemotherapy for osteosarcoma usually causes side effects such as nausea, hair loss, heart toxicity and myelosuppression, and leads to the development of acquired resistance to the cytotoxic drug in certain heterogeneous survival cells (22). Findings of recent studies have indicated that even with intensified chemotherapy of cisplatin and doxorubicin, progression-free and overall survival of osteosarcoma patients was not significantly increased compared with patients who received conventional chemotherapy (23).

The induction of angiogenesis by malignant cells has been shown to play a pivotal role in the process of tumor growth (24). In osteosarcoma, the extent of angiogenesis appears to be crucial for prognosis and survival (25-27). Inhibition of angiogenesis is, therefore, a promising method to arrest tumor growth and prevent metastasis. Previous studies have shown that activation of angiogenic activity following the resection of osteosarcoma tumors increases the progression of pulmonary metastasis (28). Using endostatin-coding plasmid or adenovirus encoding endostatin expression vector, results of various studies $(29,30)$ have shown that endostatin may inhibit 
Table II. Combination treatment of rh-endostatin with adriamycin FTW ${ }^{\mathrm{a}}$ relative to controls.

\begin{tabular}{|c|c|c|c|c|c|}
\hline \multirow[b]{2}{*}{ Dose } & \multirow[b]{2}{*}{ Rh-endostatin } & \multirow[b]{2}{*}{ Adriamycin } & \multicolumn{2}{|c|}{ Combination treatment } & \multirow{2}{*}{$\frac{\text { Ratio }}{\text { Expected/Observed }^{\mathrm{d}}}$} \\
\hline & & & Expected $^{\mathrm{b}}$ & Observed $^{c}$ & \\
\hline Low-dose & 0.75 & 0.71 & 0.53 & 0.57 & 0.93 \\
\hline High-dose & 0.66 & 0.71 & 0.47 & 0.35 & 1.34 \\
\hline
\end{tabular}

${ }^{\mathrm{a}} \mathrm{FTW}$, fractional tumor weight (mean tumor weight experimental)/(mean tumor weight control). ${ }^{\mathrm{b}}(\mathrm{Mean}$ FTW of rh-endostatin) $\mathrm{x}$ (mean FTW of the remaining experimental groups). ${ }^{\mathrm{c}}$ Observed combination treatment group FTW. ${ }^{\mathrm{d}}$ Obtained by dividing the expected FTW by the observed FTW. A ratio of $>1$ indicates a synergistic effect, a ratio of $<1$ indicates a less than additive effect.

Table III. MVD of various treatment groups.

\begin{tabular}{|c|c|c|c|c|c|c|c|}
\hline & $\operatorname{MVD}( \pm s)$ & Control & LD-RE & MD-RE & HD-RE & Adr & LD-RE and Adr \\
\hline Control & $42.4 \pm 6.0$ & & & & & & \\
\hline LD-RE ${ }^{a}$ & $33.7 \pm 2.7$ & $8.7^{* *}$ & & & & & \\
\hline MD-RE ${ }^{b}$ & $25.0 \pm 3.0$ & $17.4^{* *}$ & $8.7^{* *}$ & & & & \\
\hline $\mathrm{HD}-\mathrm{RE}^{\mathrm{c}}$ & $21.0 \pm 2.9$ & $21.3^{* *}$ & $12.6^{* *}$ & $3.9^{*}$ & & & \\
\hline$A d r^{d}$ & $38.0 \pm 3.1$ & $4.4^{* *}$ & $-4.3^{*}$ & $-13.0^{* *}$ & $-16.9^{* *}$ & & \\
\hline LD-RE and Adr & $35.7 \pm 2.1$ & $6.7^{* *}$ & -2.0 & $-10.7^{* *}$ & $-14.6^{* *}$ & 1.97 & \\
\hline HD-RE and Adr & $21.1 \pm 1.5$ & $21.2^{* *}$ & $12.6^{* *}$ & 3.8 & -0.6 & $16.9^{* *}$ & $14.6^{* *}$ \\
\hline
\end{tabular}

MVD, microvessel density; LD-RE, low-dose rh-endostatin; MD-RE, middle-dose rh-endostatin; HD-RE, high-dose rh-endostatin and Adr, adriamycin. ${ }^{*} \mathrm{p}<0.05$ and ${ }^{* *} \mathrm{p}<0.01$.

Table IV. AI of various treatment groups.

\begin{tabular}{|c|c|c|c|c|c|c|c|}
\hline & $\operatorname{MVD}( \pm \mathrm{s})$ & Control & LD-RE & MD-RE & HD-RE & Adr & LD-RE and Adr \\
\hline Control & $1.1 \pm 0.1$ & & & & & & \\
\hline LD-RE & $1.2 \pm 0.2$ & 0.1 & & & & & \\
\hline MD-RE & $1.4 \pm 0.3$ & 0.4 & 0.2 & & & & \\
\hline HD-RE & $1.6 \pm 0.2$ & $0.6^{* *}$ & $0.5^{*}$ & 0.2 & & & \\
\hline Adr & $2.4 \pm 0.2$ & $1.3^{* *}$ & $1.2^{* *}$ & $0.9^{* *}$ & $0.7^{* *}$ & & \\
\hline LD-RE and Adr & $2.2 \pm 0.1$ & $1.2^{* *}$ & $1.0^{* *}$ & $0.8^{* *}$ & $0.6^{* *}$ & -0.1 & \\
\hline HD-RE and Adr & $3.1 \pm 0.4$ & $2.1^{* *}$ & $1.9^{* *}$ & $1.7^{* *}$ & $1.5^{* *}$ & $0.8^{*}$ & $0.9^{*}$ \\
\hline
\end{tabular}

MVD, microvessel density; LD-RE, low-dose rh-endostatin; MD-RE, middle-dose rh-endostatin; HD-RE, high-dose rh-endostatin and Adr, adriamycin. ${ }^{*} \mathrm{p}<0.05$ and ${ }^{* *} \mathrm{p}<0.01$.

osteosarcoma growth in vivo. In this study, for the first time, a combination treatment of rh-endostatin with adriamycin, the earliest confirmed effective agent against osteosarcoma, was explored in human osteosarcoma in vivo.

Results of the present study show that IP-administered rh-endostatin alone had a significant anti-osteosarcoma effect. Furthermore, the addition of rh-endostatin to chemotherapy adriamycin was found to cause synergistic antitumor activity compared with chemotherapy alone. Finally, combination treatment results showed a significantly higher AI than chemotherapy alone, but without a significantly lower MVD than the rh-endostatin treatment.
In this study, compared with mono-chemotherapeutic regimens, the synergistic antitumor effect of combined antiangiogenic and chemotherapeutic agents was observed, suggesting that the combination was more effective than the sum of the effects of the monotherapies. These data, therefore, provide validation for the rationale of a combination strategy, i.e., targeting both tumor and endothelial cells to improve the treatment of osteosarcoma. The success of the combination therapy may be due to a number of factors. First, conventional chemotherapy against osteosarcoma leads to episodic application of a cytotoxic drug near the maximum tolerated doses, interrupted by a period of rest (31). During 
this period, osteosarcoma cells that survive chemotherapy may recover and proliferate. However, tumors may remain in a microscopic state of dormancy in the presence of the antiangiogenic agent (11). Second, although MVD is significantly decreased, anti-angiogenic agents tend to increase the perfusion of blood vessels, which in turn improves local anticancer drug perfusion (32). If higher adriamycin perfusion were to be achieved with an antiangiogenic drug, then the reason for the combination treatment group showing a significantly higher AI than the chemotherapy group becomes evident. Third, tumor vessels are structurally and functionally abnormal, and significantly different from normal blood vessels (33). Drug delivery, therefore, is impaired by decreased blood flow due to vascular abnormalities. Results indicate that antiangiogenic therapies may produce a sort of 'vasculature normalization' effect and improve the delivery of other therapeutics (34).

This study focuses on observing combination treatment in vivo. However, it is feasible that rh-endostatin also affects the behavior of osteosarcoma cells. In our previous study (35), we reported that rh-endostatin does not affect the proliferation of osteosarcoma cells, whether or not this occurs in the presence of adriamycin.

In conclusion, rh-endostatin inhibits the growth of osteosarcoma in a mouse model. A combination therapy of antiangiogenic and conventional chemotherapy may result in synergistic antitumor activity against osteosarcoma. Thus, the preclinical evaluation of this combination treatment appears highly attractive for clinical research. Subsequently, based on these findings, clinical trials are currently underway to determine the efficacy of this combination strategy.

\section{Acknowledgements}

The authors would like to thank Professor Yihai Cao for the critical analysis and helpful suggestions on this manuscript.

\section{References}

1. Bielack SS, Carrle D, Hardes J, Schuck A and Paulussen M: Bone tumors in adolescents and young adults. Curr Treat Options Oncol 9: 67-80, 2008.

2. Link MP, Goorin AM, Miser AW, et al: The effect of adjuvant chemotherapy on relapse-free survival in patients with osteosarcoma of the extremity. N Engl J Med 314: 1600-1606, 1986.

3. Bacci G, Longhi A, Fagioli F, Briccoli A, Versari M and Picci P: Adjuvant and neoadjuvant chemotherapy for osteosarcoma of the extremities: 27 year experience at Rizzoli Institute, Italy. Eur J Cancer 41: 2836-2845, 2005.

4. Chou AJ, Geller DS and Gorlick R: Therapy for osteosarcoma: where do we go from here? Paediatr Drugs 10: 315-327, 2008.

5. Rosen G, Marcove RC, Caparros B, Nirenberg A, Kosloff C and Huvos AG: Primary osteogenic sarcoma: the rationale for preoperative chemotherapy and delayed surgery. Cancer 43: 2163-2177, 1979.

6. Norman Jaffe: Recent advances in the chemotherapy of metastatic osteogenic sarcoma. Cancer 30: 1627-1631, 1972.

7. Niu XH, Cai YB, Zhang Q, Hao L and Ding Y: Long-term results of combined therapy for primary osteosarcoma in extremities of 189 cases. Zhonghua Wai Ke Za Zhi 43: 1576-1579, 2005.

8. Carrle D and Bielack SS: Current strategies of chemotherapy in osteosarcoma. Int Orthop 30: 445-451, 2006.

9. Daw NC, Billups CA, Rodriguez-Galindo C, et al: Metastatic osteosarcoma. Cancer 106: 403-412, 2006.

10. Ruegg C and Mutter N: Anti-angiogenic therapies in cancer: achievements and open questions. Bull Cancer 94: 753-762, 2007.
11. O'Reilly MS, Holmgren L, Chen C and Folkman J: Angiostatin induces and sustains dormancy of human primary tumors in mice. Nat Med 2: 689-692, 1996.

12. Folkman J: Antiangiogenesis in cancer therapy - endostatin and its mechanisms of action. Exp Cell Res 312: 594-607, 2006.

13. Balasubramanian L and Evens AM: Targeting angiogenesis for the treatment of sarcoma. Curr Opin Oncol 18: 354-359, 2006.

14. Carmeliet P and Jain RK: Angiogenesis in cancer and other diseases. Nature 407: 249-257, 2000.

15. Los $M$ and Voest EE: The potential role of antivascular therapy in the adjuvant and neoadjuvant treatment of cancer. Semin Oncol 28: 93-105, 2001

16. Bozec A, Lassalle S, Gugenheim J, et al: Enhanced tumour antiangiogenic effects when combining gefitinib with the antivascular agent ZD6126. Br J Cancer 95: 722-728, 2006.

17. Kato T, Sato K, Kakinuma H and Matsuda Y: Enhanced suppression of tumor growth by combination of angiogenesis inhibitor O-(chloroacetyl-carbamoyl) fumagillol (TNP-470) and cytotoxic agents in mice. Cancer Res 54: 5143-5147, 1994.

18. te Velde EA, Vogten JM, Gebbink MF, van Gorp JM, Voest EE and Borel Rinkes IH: Enhanced antitumour efficacy by combining conventional chemotherapy with angiostatin or endostatin in a liver metastasis model. Br J Surg 89: 1302-1309, 2002.

19. Weidner N: Current pathologic methods for measuring intratumoral microvessel density within breast carcinoma and other solid tumors. Breast Cancer Res Treat 36: 169-180, 1995.

20. Hockel M, Schlenger K, Hockel S and Vaupel P: Hypoxic cervical cancers with low apoptotic index are highly aggressive. Cancer Res 59: 4525-4528, 1999

21. Yokoyama Y, Dhanabal M, Griffioen AW, Sukhatme VP and RamakrishnanS: Synergy between angiostatin and endostatin: inhibition of ovarian cancer growth. Cancer Res 60: 2190-2196, 2000.

22. Hanahan D, Bergers G and Bergsland E: Less is more, regularly: metronomic dosing of cytotoxic drugs can target tumor angiogenesis in mice. J Clin Invest 105: 1045-1047, 2000.

23. Lewis IJ, Nooij MA, Whelan J, et al: Improvement in histologic response but not survival in osteosarcoma patients treated with intensified chemotherapy: a randomized phase III trial of the European Osteosarcoma Intergroup. J Natl Cancer Inst 99: 112-128, 2007.

24. Alvarez AA, Krigman HR, Whitaker RS, Dodge RK and Rodriguez GC: The prognostic significance of angiogenesis in epithelial ovarian carcinoma. Clin Cancer Res 5: 587-591, 1999.

25. Kreuter M, Bieker R, Bielack SS, et al: Prognostic relevance of increased angiogenesis in osteosarcoma. Clin Cancer Res 10: 8531-8537, 2004.

26. Mikulic D, Ilic I, Cepulic M, et al: Tumor angiogenesis and outcome in osteosarcoma. Pediatr Hematol Oncol 21: 611-619, 2004.

27. Kaya M, Wada T, Akatsuka T, et al: Vascular endothelial growth factor expression in untreated osteosarcoma is predictive of pulmonary metastasis and poor prognosis. Clin Cancer Res 6: $572-577,2000$.

28. Tsunemi T, Nagoya S, Kaya M, et al: Postoperative progression of pulmonary metastasis in osteosarcoma. Clin Orthop Relat Res 159-166, 2003.

29. Kaya M, Wada T, Nagoya S and Yamashita T: Prevention of postoperative progression of pulmonary metastases in osteosarcoma by antiangiogenic therapy using endostatin. J Orthop Sci 12: 562-567, 2007.

30. Dutour A, Monteil J, Paraf F, et al: Endostatin cDNA/cationic liposome complexes as a promising therapy to prevent lung metastases in osteosarcoma: study in a human-like rat orthotopic tumor. Mol Ther 11: 311-319, 2005.

31. Eilber FR and Rosen G: Adjuvant chemotherapy for osteosarcoma. Semin Oncol 16: 312-322, 1989.

32. Wildiers H, Guetens G, De Boeck G, et al: Effect of antivascular endothelial growth factor treatment on the intratumoral uptake of CPT-11. Br J Cancer 88: 1979-1986, 2003.

33. Hagendoorn J, Tong R, Fukumura D, et al: Onset of abnormal blood and lymphatic vessel function and interstitial hypertension in early stages of carcinogenesis. Cancer Res 66: 3360-3364, 2006.

34. Jain RK: Normalizing tumor vasculature with anti-angiogenic therapy: a new paradigm for combination therapy. Nat Med 7: 987-989, 2001

35. Xu HR, Tian W, Niu XH, et al: In vitro study of recombinant human endostatin in combination with adriamycin on human osteosarcoma cell OS-732. Chin J Bone Tumor Bone Dis 7: 129-132, 2008. 